\title{
Optical Sensor Assembly on Knee Brace for Continuous Knee Monitoring Application
}

\author{
G.M. Salim1, and M.A. Zawawi 1 \\ IFaculty of Electrical and Electronics Engineering Technology, Universiti Malaysia Pahang, 26600 Pekan, Pahang \\ ghassan.m.a@ieee.org; mohdanwar@ump.edu.my
}

\begin{abstract}
Knee joint is an important part of human body. People with poor knee condition generally have limited physical movement, rendering to mental stress and agony. Knee pain can be categorized into three groups, known as acute injury, chronic injury and medical condition. Current technology to support the knee diagnosis and treatment procedures are limited to the use of manual goniometer, $x$-ray and magnetic resonance imaging (MRI). Alternative devices with continuous measurement capability for knee monitoring are minimum at this time, mainly due to the difficulties to cover the wide angle of the knee flexion. $X$-ray and MRI technologies are useful to have some insight on the knee problem, but they are not applicable for continuous monitoring. Aside from being expensive for general use of MRI, $x$-ray on the other hand can cause short-term side effects due to radiation exposure. The aim of this paper is to demonstrate the use of optical sensor integrated with mechanical gear system as a knee monitoring device. A plastic compartment, made by using 3D printer is used to place the sensor and the gear system. The design of the overall device allows direct attachment on a knee brace for easy placement on the knee. Based on current study, the proposed sensor has a range of motion between $0 \mathrm{deg}$. to $160 \mathrm{deg}$., $0.08 \mathrm{deg}$. resolution as well as support continuous monitoring of the knee. The sensor performance has been demonstrated for gait motion, ascending and descending stairs, sit-to-stand movement and maximum knee flexion applications.
\end{abstract}

\subsection{INTRODUCTION}

Knee joint is an important part of human body which plays an important role to carry the human weight during daily activities. However, there is one common problem related to the knee which is commonly known as knee osteoarthritis (OA). In other words, it is a knee pain that is mainly due to wear and tear of the knee joint [1]. It is most common among elderly people, but middle-aged people are also prone to the same problem due to various factors such as obesity, accident and knee injuries related to knee strains, knee dislocations, knee fractures, and meniscus (cartilage) tears because of daily activities (e.g. job background, rough sport). Based on several studies, people who have history of diabetes, cancer, or cardiovascular disease are also considered as the major risk factor for osteoarthritis [2].

People who suffer from knee osteoarthritis generally show some common clinical symptoms include i) knee pain that is rapidly worsening after a few activities, ii) poor knee flexibility with/without swollen knee, and iii) continuing pain after a long period of sitting or resting [2]. Although different knee problems can potentially show similar symptoms as described earlier, further diagnosis is required to verify the source of the knee pain. Knee problem frequently diagnosed by history and physical examinations, including the use of an X-ray, MRI and arthroscopy [3]. The available technologies that are commonly used for knee problem diagnosis are summarized here. X-ray can be used to help the physician to view the cartilage, joint space and bone spurs so that the source of pain can be identified. MRI provides better images of the cartilage and knee joint to detect early abnormalities associated to osteoarthritis. Finally, arthroscopy is a procedure that involves surgical technique using a camera. It is inserted in the affected joint to visualize the damage caused to the joint by the OA [4].

Treatment of OA and knee injuries are quite different from each other. For knee injuries treatment, it depends on the seriousness of the injury. One popular therapy for knee is a list of activities involving rest, ice, compression and elevation. Besides that, some knee problems require physical therapy, immobilization or surgery. In some cases, the use of medicines to strengthen knee bones is required in treating and preventing fractures of the bones. Although a diagnosis of osteoporosis is based on the results of the bone density scan, the treatment is also based on other factors.

Two common approaches involved in knee treatments are manual therapy and exercise therapy [5]. Manual therapy for the knee is as a clinical approach that use specific hands-on techniques such as manipulation and mobilization to diagnose and treat soft tissues and joint structures. The aim of manual therapy is to improve range of motion of the knee, and to help recover the knee function. In contrast, exercise therapy is the process to implement simple exercises and stretches to the affected knee joint as part of the procedure to improve the knee condition. For both approaches, no device has clearly demonstrated the capability to measure full range knee movement between 0 deg. up to $160 \mathrm{deg}$. as well as can provide continuous data for clinical application. The limited number of suitable device for knee monitoring application has led the physician and physiotherapist to rely on manual goniometer.

To help health community related to knee treatment, we proposed a novel knee monitoring device based on optical sensor technology that includes the use of high intensity LED, linear array photodiode sensor, a set of rotational gear and rack shaft that is installed inside a plastic compartment as well as the required driver for the input and output components of the optical sensor. The output reading from the sensor is obtained based on the pixel detection along the photodiode array and then translated into its respective angle (in degree). The detection angle from the sensor is directly applied to represent the movement of the knee via the rotational movement of a knee brace. The use of knee brace in this application is important to support the knee joint recovery process and to allow a consistent sensor position on the knee during monitoring process. 


\subsection{DEVELOPMENT OF OPTICAL SENSOR FOR KNEE MONITORING}

The initial stage of the optical sensor presented here has been introduced in our previous publications [6,7]. The mathematical estimation of several parameter values required for the sensor fabrication, including gear diameter of the rotating part inside the sensor, the maximum translation of the light source along the photodiode array and the maximum range of angle detection using different parameter values are available in [6]. Based on this study, the proposed optical sensor can detect an angle range between $0 \mathrm{deg}$. to $165 \mathrm{deg}$. which is sufficient for most knee movement application such as general gait motion, ascending/descending stairs motion, sit-to-stand movement and maximum knee flexion activities. Typical range of motion for different knee assessment and/or exercise are presented in Table 1.

Table 1: Typical knee range of motion for different activities

\begin{tabular}{|c|l|c|c|}
\hline No & Optical Sensor type & Range of motion (deg.) & Ref. \\
\hline 1 & Gait (i.e. : level walking) & $57-69 \mathrm{deg}$. & {$[\mathbf{8}]$} \\
\hline 2 & Ascending/descending stairs & $83-99 \mathrm{deg}$. & {$[\mathbf{9 ]}$} \\
\hline 3 & Sit to stand from a chair & $84-120 \mathrm{deg}$. & {$[\mathbf{1 0}]$} \\
\hline 4 & Maximum knee flexion & Max $155 \mathrm{deg}$. & {$[\mathbf{1 1}]$} \\
\hline
\end{tabular}

The details of components involved in the optical sensor assembly are also provided in here [7]. These include the type of light emitting diode (green LED as light source), linear array photodiode (as light detector), associated circuits assembly, rotational part of the sensor such as gear, rack shaft and the mechanical assemble that encased the whole components of the sensor. The overall sensor dimension before being attached to the knee braces is $(12 \times 8 \times 3) \mathrm{cm}(\mathrm{L}$ $\mathrm{x}$ W $\mathrm{x}$ D) at 150 grams of weight, not including the electronic circuitry that are separate placed (not on knee braces).

The overall assemble of the sensor before and after being attached onto the knee brace is shown in Figure 1, 2 and 3, respectively.
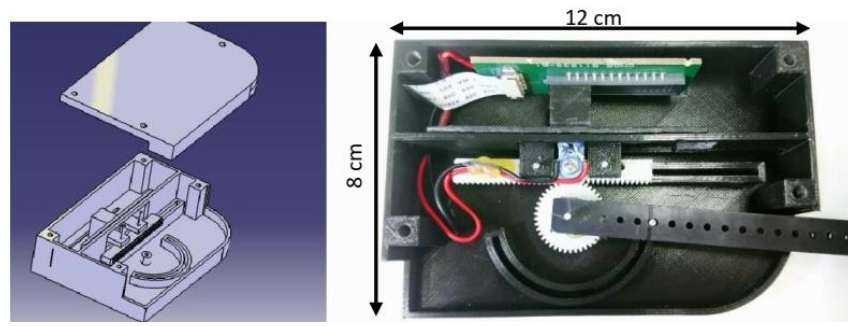

Figure 1: Inside components of the optical sensor [7]

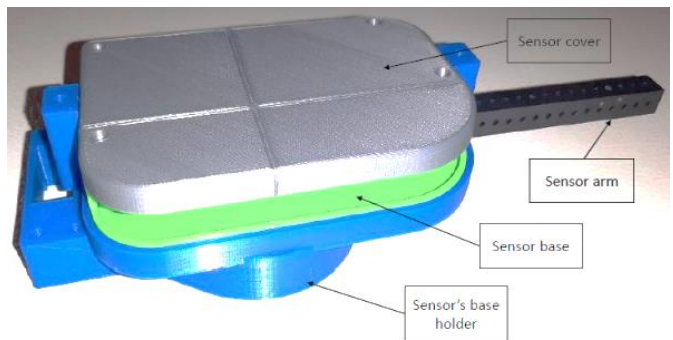

Figure 2: Optical sensor assembly inside the mechanical compartment

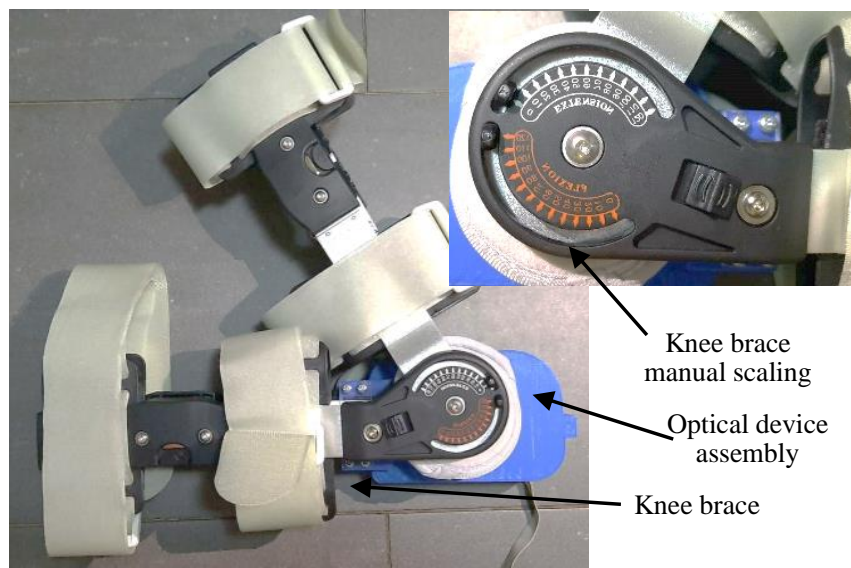

Figure 3: Optical sensor attachment on a knee brace. The knee brace can rotate to maximum $120 \mathrm{deg}$. from a straight position

The knee brace is designed to assess stability of the knee, decrease potential injuries to the knee, and help maintain the athletic performance for sport's personnel [12]. It is considered an essential component during the rehabilitation process, as it improves knee ligaments stability and motion's axial alignment. Knee braces are normally applied for three main reasons that are to prevent an occurrence of knee injury, provide stability for weak knees, and control the alignment of knee joint motion [13]. Patients who used knee braces reported improvements of knee joint stability, pain reduction, and enhanced mobility performance. For these reasons, knee brace is included in our overall sensor setup to assess in measuring the knee joint angle during continuous movement while insuring a reliable angular motion on the exact knee joint central of rotation concentrated on the frontal plane only without having to face error from angular movement on the sagittal plane.

\subsection{COMPARISON BETWEEN DIFFERENT SENSORS FOR KNEE MONITORING APPLICATION}

The proposed optical sensor for knee monitoring application is compared to several type of optical-based devices to illustrate the advantage of this sensing approach. The aim of this comparison is to identify the most suitable device that can be applied for different knee activities, especially those are typically being implemented by most physiotherapist during assessment and/or physical therapy of the patients. Despite many development of sensor technology for different human health monitoring applications, most clinics or therapy centres still rely on the manual goniometer [14] to measure the movement range of human joints, such as knee, elbow, shoulder, fingers and hip. One limitation of using the goniometer is that the measurement reliability depends on the human experience, so human error is unavoidable. Moreover, previous study in [15] stated that involuntary activity of the peroneal muscle group during knee joint movement can influence the measurement results. These problems explain the need for alternative devices for knee monitoring application.

One of the knee measurement device produced before is called optical-based curvature sensor. This sensor applied the concept of light intensity variation to represent the knee joint angle due to knee movement. As the knee movement angle is increased, more light will escape the fiber core especially in the area of teeth-like sensitive zone along the fiber. The 
author claimed that this device is low cost, non-invasive, and not affected to external electromagnetic interferences [16], With an accuracy of $\pm 1 \mathrm{deg}$. and along with a resolution of 1 deg., it is found that the device has some limitations related to poor reliable measurement results at low angle (e.g. between the 0-25 deg.) and inconsistent output reading at high angle (e.g. more than $120 \mathrm{deg}$.). It was believed that the reason is due to the critical condition (e.g. prone to crack) of the fiber at the sensitive zone during extra bending.

Another alternative device for human joint measurement is called optical fiber-based bending sensor using optical fiber tilting loss effect. This approach has been applied to measure the spine flexion and extension in front, back and side directions. The device is designed based on two separated fibers, each connected to a light source and photodetector. The tilting angle between these fibers is used to represent the joint angle. The author claimed that the device has high accuracy, good resolution, lightweight device, non-invasive, and immune to electromagnetic interferences. Based on our review, the accuracy of the device is $0.32 \mathrm{deg}$. and at a resolution of $0.15 \mathrm{deg}$. The main reason why this technique is not suitable for knee application is that the available detection range is only between $-25 \mathrm{deg}$. to $25 \mathrm{deg}$. [17].

There is another optical based device that has been introduced to measure the joints movement at the palm, wrist and elbow. The fiber Bragg grating (FBG)-based sensor is placed inside a special silica gel before the whole sensor is attached on a stretchable garment. It can be noticed that the device has a simple construction and easy to be applied to different body areas but the working range of the device is limited between $0 \mathrm{deg}$. to $60 \mathrm{deg}$., which is clearly insufficient for knee application [18]. In addition, most optical device based on FBG requires the use of advanced and expensive spectrometer to detect the wavelength changes of the light source.

The next optical fiber device has been applied to measure the knee joint movement and is known as optical goniometer. The joint movement is detected based on the change of light intensity of a laser beam propagating inside a single-mode optical fiber. The polarization of the light is varied by the rotation of the treated area of the fiber as the knee joint movement is triggered. Although the device has an accuracy of 0.1 deg. and resolution of 0.01 deg., the maximum application range of the sensor at $90 \mathrm{deg}$. is not quite sufficient for full range knee detection. In addition to this, the device setup consists of expensive laser input, linear polarizer, and stress induced birefringence polarization controller, rendering to a complicated system to be applied as portable monitoring device [19].

The last device is based on the use of a so-called plastic optical fiber curvature sensor that involves a side-polished fiber, and is mainly used to detect the elbow movement. The size of the polished area (sensitive zone) in terms of its length and depth was at $14 \mathrm{~mm}$ and $0.6 \mathrm{~mm}$. Considering the diameter of the Polymethyl Methacrylate (PMMA) fiber is $0.98 \mathrm{~mm}$, the polished area is likely to break at very high bending angle (i.e. $62 \%$ of the fiber wall thickness was cut). The authors reported that this device is compact, lightweight, flexible and immune to electromagnetic interference, but it has limited measurement range between $0 \mathrm{deg}$. and $90 \mathrm{deg}$. and poor accuracy of $\pm 3.71 \mathrm{deg}$. [20].

Typically, the knee joint can move in flexion direction between $0^{\circ}-140^{\circ}$, where the range depends on the type of activities (e.g. gait, sit-to-stand or supine) conducted by the patient. Table 2 summarized the range of motion, sensor resolution and original intended application of each device discussed in this section. As a summary, current optical-based sensors are not entirely suitable for all type of knee movement including gait, sit-to-stand position, ascending and descending stairs and full knee flexion.

Table 2: Summary of comparison between several opticalbased sensors for joint movement applications

\begin{tabular}{|c|c|c|c|c|c|}
\hline No & Sensor Type & $\begin{array}{l}\text { ROM* } \\
\text { (deg.) }\end{array}$ & $\begin{array}{c}\text { Resolution } \\
\text { (deg.) }\end{array}$ & $\begin{array}{c}\text { Intended } \\
\text { Application }\end{array}$ & Ref. \\
\hline 1 & $\begin{array}{l}\text { Manual } \\
\text { goniometer }\end{array}$ & $\begin{array}{l}0 \text { to } \\
180\end{array}$ & $1 \mathrm{deg}$. & General & [14] \\
\hline 2 & $\begin{array}{l}\text { Optical curvature } \\
\text { sensor (with } \\
\text { sensitive zone) }\end{array}$ & 0 to 25 & $1 \mathrm{deg}$. & Knee & [16] \\
\hline 3 & $\begin{array}{l}\text { Optical fiber } \\
\text { bending sensor }\end{array}$ & $\begin{array}{c}-22 \text { to } \\
+22\end{array}$ & $0.15 \mathrm{deg}$. & Spine & [17] \\
\hline 4 & $\begin{array}{l}\text { Fiber Bragg } \\
\text { grating }\end{array}$ & 0 to 60 & N/A & $\begin{array}{l}\text { Wrist, } \\
\text { elbow }\end{array}$ & [18] \\
\hline 5 & $\begin{array}{l}\text { Optical } \\
\text { goniometer }\end{array}$ & 0 to 90 & $0.1 \mathrm{deg}$. & Knee & [19] \\
\hline 6 & $\begin{array}{l}\text { Plastic fiber with } \\
\text { side-polished }\end{array}$ & 0 to 90 & $3.71 \mathrm{deg}$. & Elbow & [20] \\
\hline 7 & $\begin{array}{l}\text { Optical sensor } \\
\text { with photodiode } \\
\text { array }\end{array}$ & $\begin{array}{l}0 \text { to } \\
160\end{array}$ & $0.08 \mathrm{deg}$. & Knee & N/A \\
\hline
\end{tabular}

* Range of motion of the respective monitoring devices.

Based on the above comparison, we proposed an improved optical sensor which is slightly optimized from our previous sensor arrangement [7] that produce measurement accuracy of $1 \mathrm{deg}$. and resolution of $0.5 \mathrm{deg}$. These changes include the modification of the gap between the light beam of the LED and the detection size of the photodiode array (considering the number of pixel of the detector). The new range of motion of the sensor is now further increased to $160 \mathrm{deg}$. with a better resolution of $0.08 \mathrm{deg}$., as demonstrated in our publication [21] in details. Moreover, the sensor still supports online data measurement with data recording of up to 2 hours.

The sensor development stage has been addressed previously in [21]. In this paper, we demonstrated the development of the state-of-the art of the optical sensor that consists of a green LED, a linear array photodiode, a plastic gear assembly, and related electronics components. To validate and demonstrate the accuracy and reliability of the proposed sensor, the sensor has been tested on an in-house test rig that was directly compared to a manual goniometer (side-by-side placement of the sensor and goniometer). This was important to allow both devices to move simultaneously on the same angle as the flexible arm of the test rig (i.e. which represent the knee flexion) was rotated. A comparison table was provided to compare the measurement results of the manual goniometer against the proposed optical device, as shown in Table 3 [21]. From the table, it is shown that the biggest error of proposed sensor measurement is only at $0.04^{\circ}$ as compared to the goniometer reading. In previous publication, no knee measurement has been carried out using the proposed optical sensor. 
Table 3: Goniometer and device comparison table [21].

\begin{tabular}{|c|c|c|}
\hline \multirow{2}{*}{$\begin{array}{l}\text { Goniometer Reading } \\
\text { (Angle in degree) }\end{array}$} & \multicolumn{2}{|c|}{ Device Reading } \\
\hline & Pixel Number & Angle in degree \\
\hline $0^{0}$ & Pixel = 1 & $0.08^{\circ}$ \\
\hline $5^{0}$ & Pixel $=63$ & $5.04^{\circ}$ \\
\hline $10^{\circ}$ & Pixel $=125$ & $10.00^{\circ}$ \\
\hline $15^{0}$ & Pixel $=188$ & $15.04^{0}$ \\
\hline $20^{\circ}$ & Pixel $=250$ & $20.00^{\circ}$ \\
\hline $25^{\circ}$ & Pixel $=313$ & $25.04^{0}$ \\
\hline $30^{\circ}$ & Pixel $=375$ & $30.00^{\circ}$ \\
\hline $35^{\circ}$ & Pixel $=438$ & $35.04^{0}$ \\
\hline $40^{\circ}$ & Pixel $=500$ & $40.00^{\circ}$ \\
\hline $45^{\circ}$ & Pixel $=563$ & $45.04^{0}$ \\
\hline $50^{\circ}$ & Pixel $=625$ & $50.00^{\circ}$ \\
\hline $55^{\circ}$ & Pixel $=688$ & $55.04^{0}$ \\
\hline $60^{\circ}$ & Pixel $=750$ & $60.00^{\circ}$ \\
\hline $65^{\circ}$ & Pixel $=813$ & $65.04^{0}$ \\
\hline $70^{\circ}$ & Pixel $=875$ & $70.00^{\circ}$ \\
\hline $75^{\circ}$ & Pixel $=938$ & $75.04^{0}$ \\
\hline $80^{\circ}$ & Pixel $=1000$ & $80.00^{\circ}$ \\
\hline $85^{\circ}$ & Pixel $=1063$ & $85.04^{0}$ \\
\hline $90^{\circ}$ & Pixel $=1125$ & $90.00^{\circ}$ \\
\hline $95^{\circ}$ & Pixel $=1188$ & $95.04^{0}$ \\
\hline $100^{\circ}$ & Pixel $=1250$ & $100.00^{\circ}$ \\
\hline $105^{\circ}$ & Pixel $=1313$ & $105.04^{\circ}$ \\
\hline $110^{\circ}$ & Pixel $=1375$ & $110.00^{\circ}$ \\
\hline $115^{\circ}$ & Pixel $=1438$ & $115.04^{\circ}$ \\
\hline $120^{\circ}$ & Pixel $=1500$ & $120.00^{\circ}$ \\
\hline $125^{\circ}$ & Pixel $=1563$ & $125.04^{\circ}$ \\
\hline $130^{\circ}$ & Pixel $=1625$ & $130.00^{\circ}$ \\
\hline $135^{\circ}$ & Pixel $=1688$ & $135.04^{\circ}$ \\
\hline $140^{\circ}$ & Pixel $=1750$ & $140.00^{\circ}$ \\
\hline $145^{\circ}$ & Pixel $=1813$ & $145.04^{\circ}$ \\
\hline $150^{\circ}$ & Pixel $=1875$ & $150.00^{\circ}$ \\
\hline $155^{\circ}$ & Pixel $=1938$ & $155.04^{0}$ \\
\hline $160^{\circ}$ & Pixel $=2000$ & $160.00^{\circ}$ \\
\hline
\end{tabular}

Figure 4 illustrates the actual reading from the sensor output using dedicated software to represent the output.
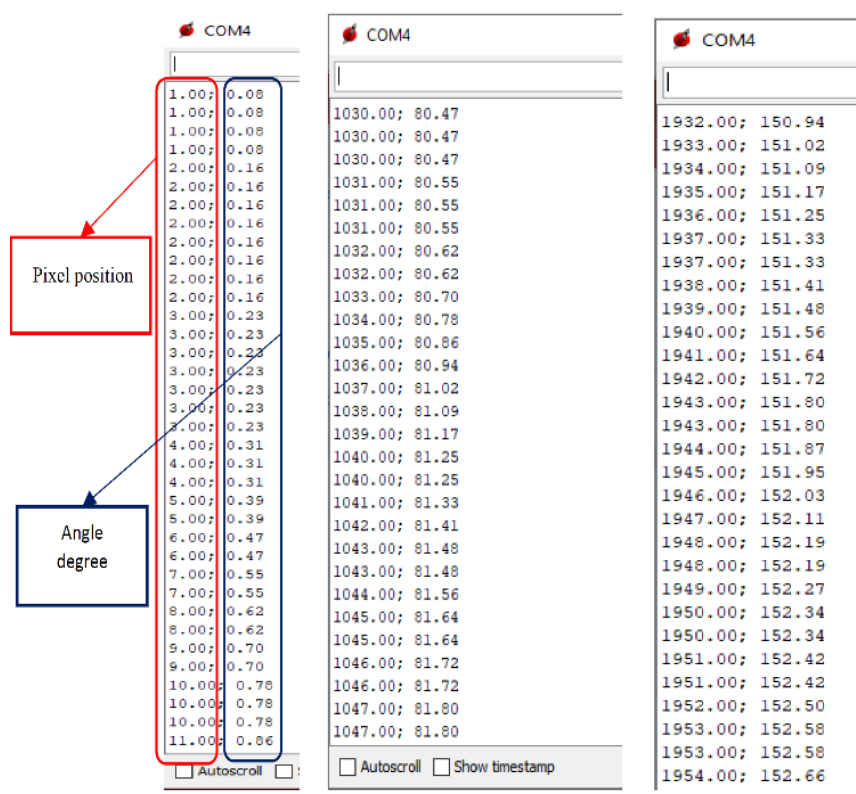

Figure 4: Sensor output in pixel number vs. angle (in deg.)

In this paper, we demonstrate the implementation of the proposed optical sensor, embedded together on a standard knee brace, to create more flexibility of sensor placement directly of the knee area. Basic knee movements, involving gait, stairs climbing, sit-to-stand movement and full knee flexion activities. More clinical test will be carried out by the authors, with appropriate guidance from local physiotherapy will be carried out to demonstrate the reliability and accuracy of the proposed device for knee monitoring application in the near future.

\subsection{RESULTS AND ANALYSIS}

The optical sensor proposed in this work is applied with the assist of mechanical rotating gear and shaft as shown in Figure 1. After the assemble process is completed, the optical sensor is directly attached on a knee brace using a mechanical clamp. This clamp allows the sensor to be removed whenever necessary if further calibration is required in the future. The knee brace along with the optical sensor set is later applied to a person knee via the stretchable straps at the thigh and calf level to allow comfortable placement of the knee brace. After that the person is instructed to do different activities as follow: 1) gait movement (walking), 2) ascending and descending stairs, 3) sit-to-stand on a chair and 4) maximum flexion of the knee during supine position. The last activity here is conducted without the use of knee brace as the maximum allowable rotation of the knee brace is only up to $120 \mathrm{deg}$.

\subsection{SENSOR PLACEMENT ON THE KNEE}

The sensor is attached on a medical grade knee brace which allows a corresponding movement as the knee joint. For the type of knee brace used in the study, the maximum rotating range of the knee brace may reach up to $120^{\circ}$, thus limits the knee joint movement measurement of any activity within the range of $120^{\circ}$. The wearable device is as shown in Figure 5 below.

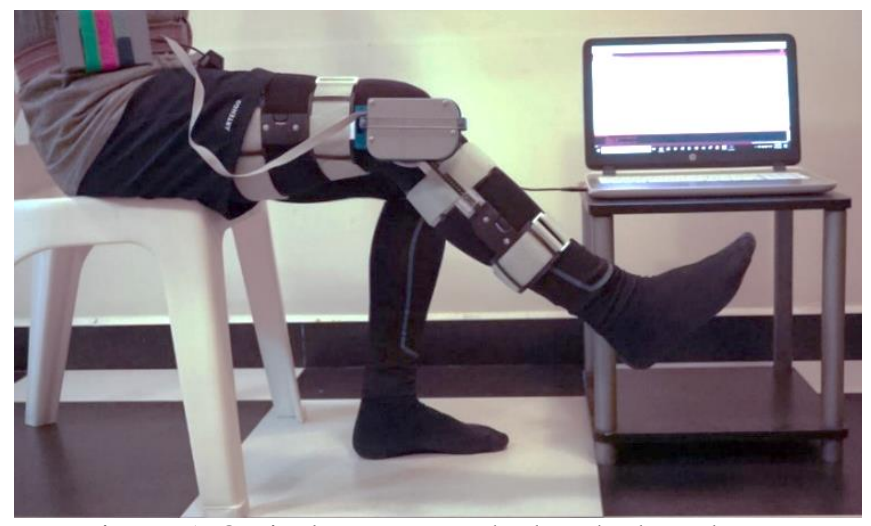

Figure 5: Optical sensor attached to the knee brace.

\subsection{SENSOR TEST ON GAIT ACTIVITY}

As stated in section 2, the maximum angular movement of the knee joint during gait for a healthy adult should ranges between 57 to $69 \mathrm{deg}$. Hence, the wearable sensor device was worn by the volunteer to demonstrate the joint angle during gait. As observed from the graph in Figure 6, the initial starting point of the subject while standing is at $5 \mathrm{deg}$., before the knee joint moves due to walking activity, where the angle of the knee joint increases to $65 \mathrm{deg}$., then the waveform drops to its initial starting point when the joint is at neutral position. However, the drop of the wave has slight fluctuation which is caused by the impact of the foot on the ground surface. The waveform keeps repeating symmetrically showing a consistent gait condition of the subject and the gait waveform's amplitude is within the range of a healthy adult's characteristics. 


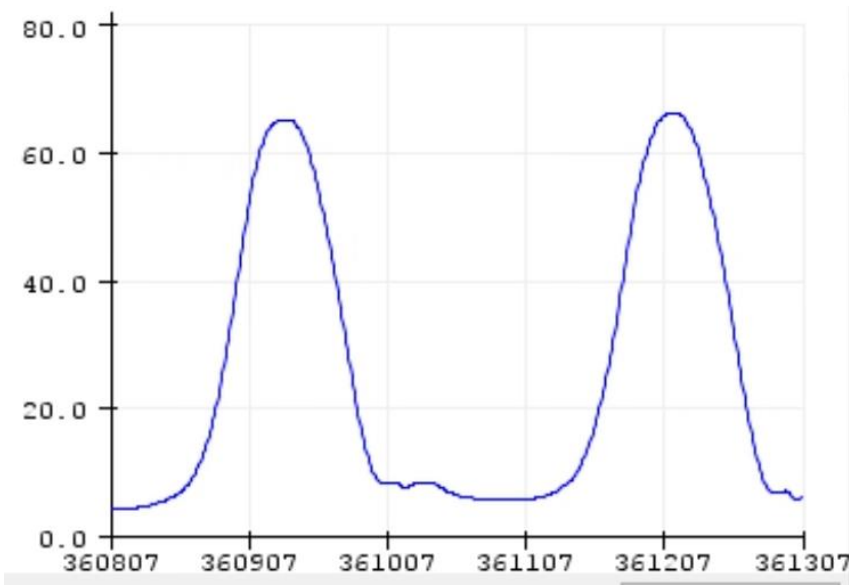

Figure 6: Sensor output measurement during gait.

\subsection{SENSOr Test ON AsCENDING AND DESCENDING StAIRS}

Ideally, bending of the knee joint during ascending is at $83^{\circ}$. Meanwhile, $90^{\circ}$ when descending the stairs. The subject under study was asked to go up and down the stairs while wearing the sensor device as shown in Figures 7.

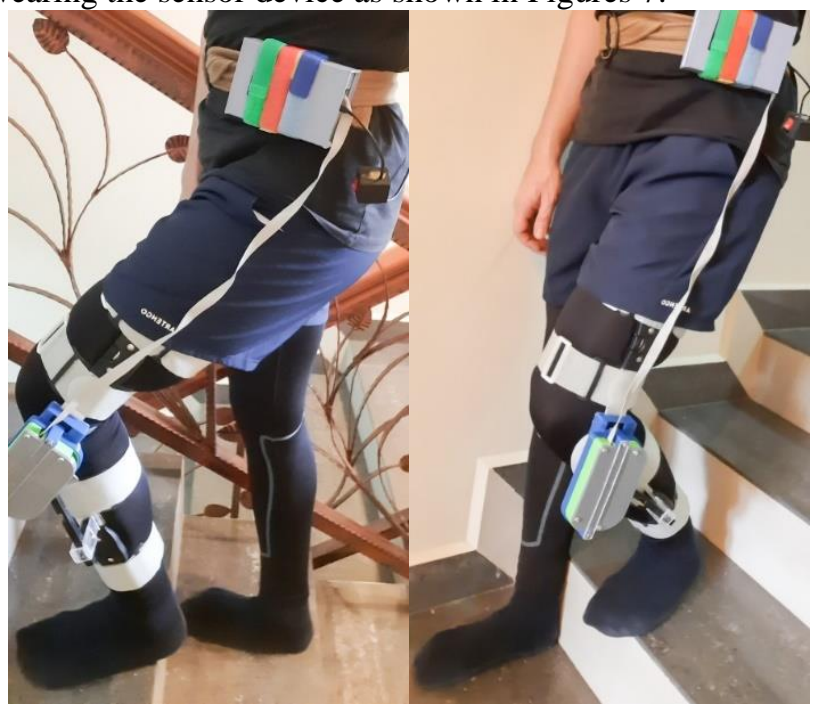

Figure 7: Ascending and descending the stairs activities.

As can be observed in Figures 7, the wearable optical sensor assessment device is applied on the subject while ascending and descending the stairs. Consequently, the sensor generated a continuous waveform display in angle degree while performing the activity as shown in Figures 8 and 9 for ascending and descending activities, respectively.

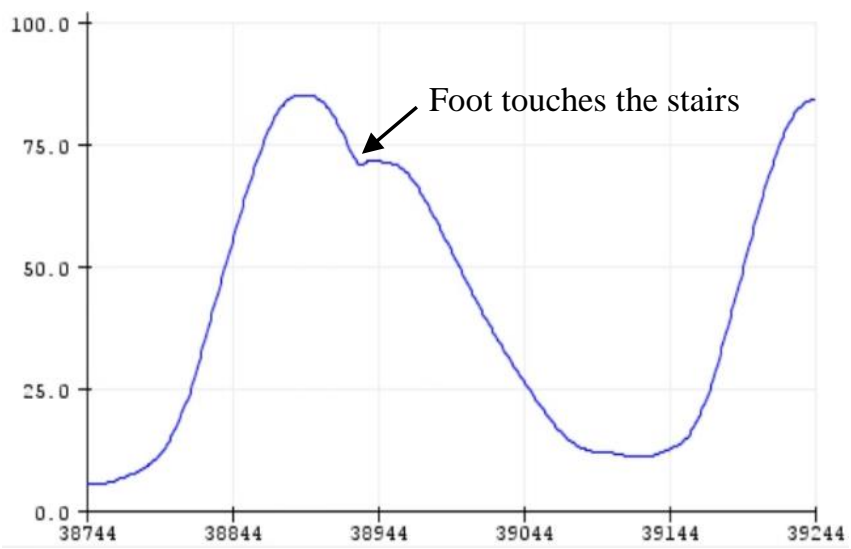

Figure 8: Sensor output measurement during ascending the stairs.

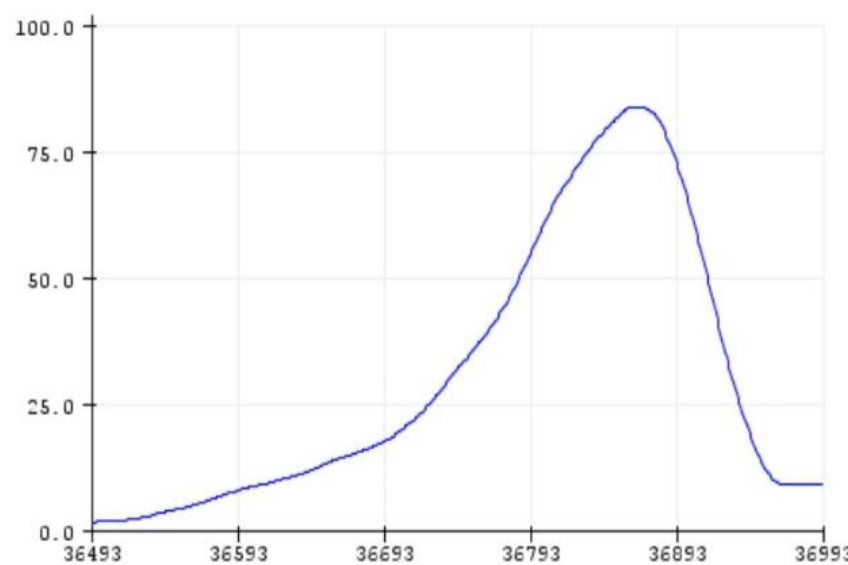

Figure 9: Sensor output measurement during descending the stairs.

As shown in Figure 8, the starting point for the subject is at $5 \mathrm{deg}$. at which the person is at a static standing positon. Then, when the subject stepped forward to climb up the staircase, the graph shows a steep incline until reaching an amplitude of $86 \mathrm{deg}$. The moment the foot of the subject becomes in contact with the floor surface, the impact force causes slight fluctuation in the output graph and then continuous to decline as the person starts to straighten the joint upon raising up the staircase until reaching $11 \mathrm{deg}$., the patient starts initiating another step forward which causes the graph to increase again forming a similar waveform repeatedly with every step taken to ascend the stairs.

Figure 9 shows a graphical output for descending the stairs activity. While moving down the stairs, the leg that moves forward to descend the stairs remains at neutral position without bending and the other leg that remains on the original position bends due to the height decline between the two steps. When the leg bends (knee joint flexes), it will then move forward to the following step on the staircase. Based on the graph, the initial starting point at $1 \mathrm{deg}$. (foot is ready to move), then as the person moves forward descending the stairs, the knee joint flexes until it reaches an angular amplitude of $87 \mathrm{deg}$. After the knee joint reaches maximum required angular flexion, the leg will then be lifted in the air moving forward to the bottom step, causing the graphical output to decline until the feet stands is static on the lower staircase. It is shown in the graph when the patient reaches the lower step, the knee joint angle is at $5 \mathrm{deg}$., and then the same waveform would repeat consistently as the person continuous descending the stairs.

These results show that the optical sensor is capable of measuring the ascending and descending the stairs activities continuously which is useful for knee monitoring application.

\subsection{SENSOR TEST ON SIT-TO-STAND FrOM A CHAIR}

In this section, we present another knee movement activity which is stand-to-sit and sit-to-stand positions. For a healthy knee condition, the movement involving sit-to-stand should not give too much difficulty for a person. However, for unhealthy knee sufferers this activity might cause discomfort to them. It is worth to mention that, complication in conducting such movement can be an indicator of degenerative muscular or ligaments health condition. In this study, the activity is carried out with the knee joint angle initially at sitting position (between $80 \mathrm{deg}$. to $120 \mathrm{deg}$.), then 
the patient is asked to stand to straight position (angle between 0 to 5 deg.). The knee positions during sit and stand are as shown in Figure 10 and 11, respectively.

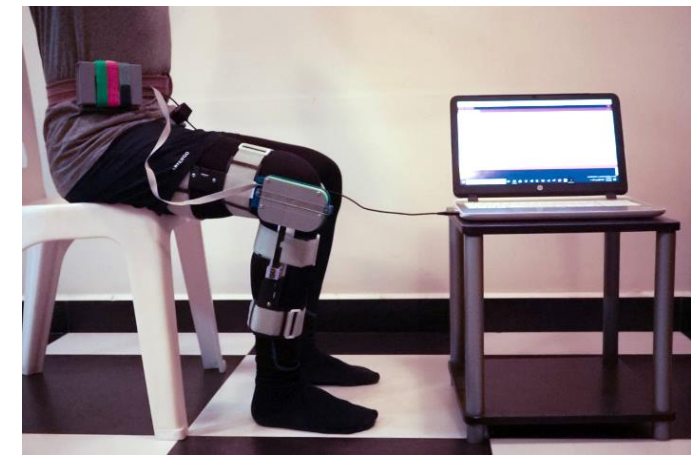

Figure 10: Subject at sitting position.

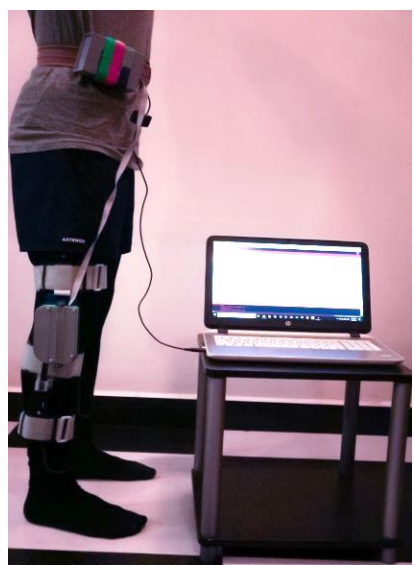

Figure 11: Subject at standing position.

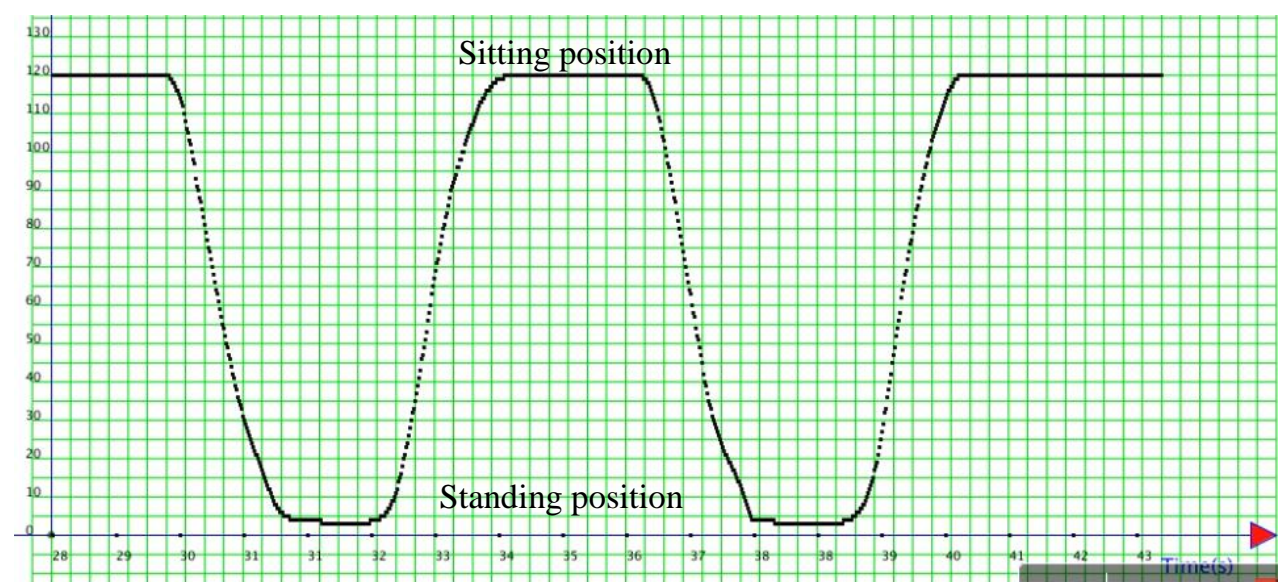

Figure 12: Sensor output measurement during sit-to-stand movement.

As can be observed in Figure 12, the graph shows an angle of $120 \mathrm{deg}$. when the subject initially seated on a chair. Later the knee angle decline to as low as $3 \mathrm{deg}$. after the subject moved to stand position. To complete the cycle, the subject was asked to seat again in the same chair, rendering an output inclination from 3 to $120 \mathrm{deg}$. The sit-to-stand movement is repeated for several times to verify the repeatability of the sensor. A consistent output waveform between each sit-tostand cycle was observed during this process, thus demonstrates a stable output reading provide by the optical sensor which makes it possible for the intended knee monitoring application.

\subsection{MAXIMUM FLEXION OF THE KNEE}

Maximum knee flexion for a healthy adult can reach up to $157 \mathrm{deg}$. when fully pressed, such as in squatting position (with heels up) [22]. Another position that requires almost maximum knee flexion is during supine position as shown in Figure 13. Although the proposed wearable optical sensor device range of measurement can reach up to $160 \mathrm{deg}$., the maximum achievable range for the device (optical sensor being attached to the knee brace) is only up to $120 \mathrm{deg}$. because the knee brace can only allow maximum rotation up to 120 deg. (refer Figure 3). For this reason, the measurement results shown in Figure 14 is obtained when the optical sensor is separately applied without the knee brace. This condition is considered acceptable because the assessment of maximum flexion of the knee can be determined under static condition, thus knee brace support is less important for this case as compared to other type of dynamic assessments (e.g. gait, sitto-stand and ascending/descending stairs).

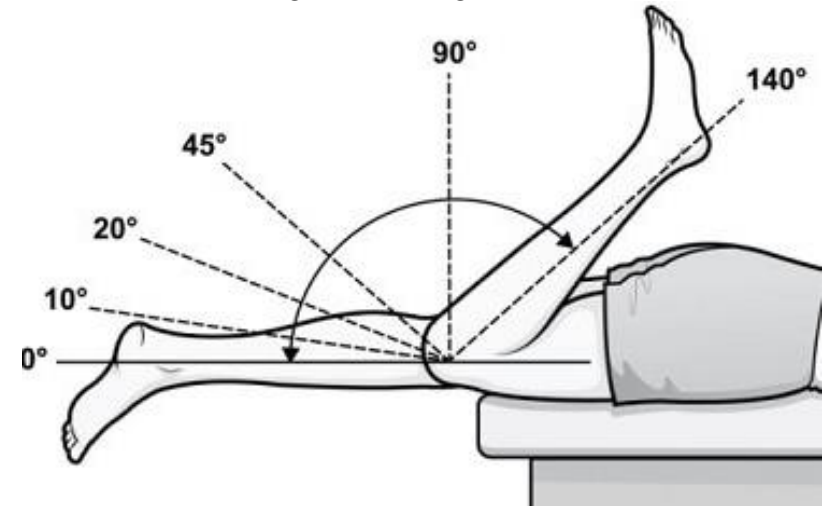

Figure 13: Maximum knee movement during supine position. 


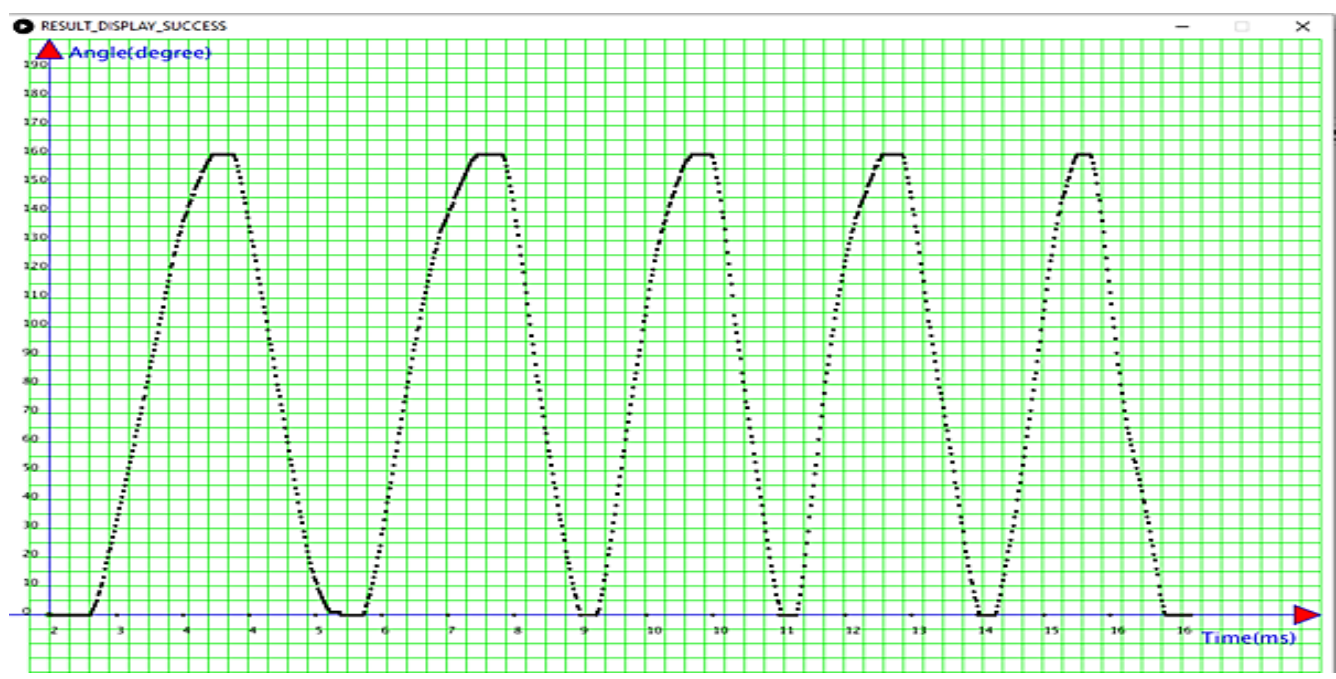

Figure 14: Maximum knee flexion graph [21].

\subsection{DeVice CliniCAl APPLICABILITY}

As discussed in this paper, different knee movement produces different range of knee flexion angles. Most optical based devices for human joint monitoring [16-20] so far can only provide a maximum range of detection of up to $90 \mathrm{deg}$. angle. This is lower than the maximum required knee angle when performing several activities such as sit-to-stand activities and full knee flexion during supine and squatting positions. Our proposed wearable optical-based knee monitoring sensor is capable of measuring an angle of up to $160 \mathrm{deg}$. as shown in Figure 14, which makes it suitable for knee monitoring application, including the activities in Table 4.

Table 4: Range of knee joint angle during

\begin{tabular}{|c|c|c|c|}
\hline Test Condition & $\begin{array}{c}\text { Min. Angle } \\
\text { (Degree) }\end{array}$ & $\begin{array}{c}\text { Max. Angle } \\
\text { (Degree) }\end{array}$ & $\begin{array}{c}\text { Proposed } \\
\text { sensor }\end{array}$ \\
\hline Gait & 0 deg. & 69 deg. & $\checkmark$ \\
\hline $\begin{array}{c}\text { Sit-to-Stand on } \\
\text { Chair }\end{array}$ & 0 deg. & 120 deg. & $\checkmark$ \\
\hline Ascending Stairs & 0 deg. & 99 deg. & $\checkmark$ \\
\hline Descending Stairs & 0 deg. & 99 deg. & $\checkmark$ \\
\hline Full flexion (supine) & 0 deg. & 140 deg. & $\checkmark$ \\
\hline $\begin{array}{c}\text { Full flexion } \\
\text { (squatting) }\end{array}$ & 0 deg. & 157 deg. & $\checkmark$ \\
\hline
\end{tabular}

\subsection{CONCLUSION}

Knee assessment is a useful procedure for patients who are in the diagnosis and treatment stages of different knee problems. Current technologies such as x-ray, MRI and manual goniometer cannot fully support the continuous monitoring of the knee, rendering limited data can be made available for respective clinical personnel. Despite a number of alternative sensors introduced by researchers, these devices do not fully support the needs of knee application, especially due to the limited range of motion of those sensors. In this paper, we demonstrate a wearable optical-based knee monitoring device, specially designed to allow direct attachment on a knee brace. The combination of the optical sensor and the knee brace made it possible to simultaneously strengthen the poor knee condition and to collect data related to the knee movement within a specific time period. The proposed optical sensor has a larger range of motion than other comparable optical-type devices, with a maximum detection angle of $160 \mathrm{deg}$. that is larger than required angle of maximum knee flexion of 157 deg.. The experimental results in this study also proved that the proposed optical sensor is suitable for clinical application covering different activities of the knee such as gait, sit-to-stand, ascending and descending stairs and maximum flexion. More field test will be carry out with the help of local physiotherapist to further validate the sensor performance.

\subsection{ACKNOWLEDGEMENT}

The authors wish to thank Universiti Malaysia Pahang (UMP) and Kementerian Pendidikan Malaysia (KPM) for the funding awarded to the author to complete this study under the national grant reference number: FRGS/1/2019/TK04/UMP/02/18 (FRGS) and RDU1901218 (UMP).

\section{REFERENCES}

[1] Zhang Y, Jordan JM. Epidemiology of osteoarthritis. Clinics in Geriatric Medicine. Aug 2010, Vol. 26(3), pp. 355-369.

[2] Altman RD, Early management of osteoarthritis, American Journal of Managed Care. Mar 2010, Vol. 16(2), pp. S41-S47.

[3] Bliddal H, Christensen R. The treatment and prevention of knee osteoarthritis: a tool for clinical decision-making. Expert Opinion on Pharmacotherapy. June 2009, Vol. 10(11), pp.1793-1804.

[4] Lespasio MJ, Piuzzi NS, Husni ME, Muschler GF, Guarino AJ, Mont MA. Knee osteoarthritis: a primer. The Permanente Journal. Sept 2017, Vol. 21, 16-183.

[5] Abbott J. H., Robertson M. C., McKenzie, J. E., Baxter G. D, Theis J., and Campbell A. J., Exercise therapy, manual therapy, or both, for osteoarthritis of the hip or knee: a factorial randomised controlled trial protocol, Trials Journal, Feb. 2009, Vol. 10(1), pp. 1-12

[6] Salim, G. M., Zawawi, M. A., Mathematical Representation of Joint Angle Measurement using Step Index Optic Fibre and Linear Array Photodiode Sensor. Journal of Physics: Conference Series, 2020, Vol. 1529 pp. 042089.

[7] Salim, G. M., Zawawi, M. A., A Novel Implementation of Knee Joint Monitoring Device using Step Index Optical Fibre and Linear Array Photodiode Sensor. Journal of Physics: Conference Series, 2020, Vol. 1529 pp. 042069.

[8] Myles C M, Rowe P J, Walker C R C and Nutton R W. Knee joint functional range of movement prior to and following total knee arthroplasty measured using flexible electrogoniometry. Gait \& Posture, 2002, Vol. 16(1), pp. 46-54.

[9] Rowe P J, Myles C M, Walker C and Nutton R. Knee joint kinematics in gait and other functional activities measured using flexible electrogoniometry: How much knee motion is sufficient for normal daily life? Gait Posture, 2000, Vol. 12(2), pp. 143-155.

[10] Tully E A, Fotoohabadi M R and Galea M P. Sagittal spine and lower limb movement during sit-to-stand in healthy young subjects. Gait Posture, 2005, Vol. 22(4), pp. 338-345. 
[11] Devers B N, Conditt M A, Jamieson M L, Driscoll M D, Noble P C and Parsley B S. Does greater knee flexion increase patient function and satisfaction after total knee arthroplasty? Journal of Arthroplasty, 2011, Vol. 26(2), pp. 178-186.

[12] Scott A. P. Knee Braces: Current Evidence and Clinical Recommendations for Their Use, American Family Physician Available online: https://www.aafp.org/afp/2000/0115/p411.html.

[13] Riskowski J. L., Mikesky A. E., Bahamonde R. E., and Burr D. B. Design and Validation of a Knee Brace with Feedback to Reduce the Rate of Loading. Journal of Biomechanical Engineering, 2009, Vol 131(8), pp. 084503 (6 pages)

[14] Trappler R., Smith E., Goldberg G., Parvizi J. and Hozack W.J. Knee Range of Motion : Can we believe the Goniometer Reading?. Orthopaedic Proceedings, Vol 91-B, SUPP_I, 6-6.

[15] Clapper M. P. and Wolf S. L. Comparison of the Reliability of the Orthoranger and the Standard Goniometer for Assessing Active Lower Extremity Range of Motion. Physical Therapy, Feb 1988, Vol. 68(2), pp. 214-218.

[16] Stupar D Z, Bajic J S, Manojlovic L M, Slankamenac M P, Joza A V, and Zivanov M B. Wearable Low-Cost System for Human Joint Movements Monitoring Based on Fibre-Optic Curvature Sensor IEEE Sensors Journal, 12(12), 2012, pp. 3424-3431.

[17] Zawawi M A, O'Keeffe S, and Lewis E. An Extrinsic Optical Fibre Bending Sensor: A Theoretical Investigation and Validation IEEE Sensors Journal, 15(9), 2015, pp. 5333-5339.

[18] Abro Z A, Yi-Fan Z, Cheng-Yu H, Lakho R A, Nan-Liang C. Development of a Smart Garment for Monitoring Body Postures based on FBG and Flex Sensing Technologies, Sensors and Actuators A, 272 , 2018, pp. 153-160.

[19] Massimiliano Donno, A New Flexible Optical Fiber Goniometer for Dynamic Angular Measurements: Application to Human Joint Movement Monitoring, IEEE Transactions on Instrumentation and Measurement, Vol. 57, No. 8, August 2008.

[20] Andressa Rezende, Polymer Optical Fiber Goniometer: A New Portable, Low Cost and Reliable Sensor for Joint Analysis. Sensors. Vol. 18(12), 2018, pp. 4293.

[21] Salim, G. M and Zawawi, M. A. Knee Monitoring Device Based on Optical Sensor Embedded in Mechanical Compartment Assembly, Optik (Elsevier). Vol. 223, Dec 2020, pp. 165546.

[22] Hemmerich A, Brown H, Smith S, Marthandam S S and Wyss U P. Hip, knee, and ankle kinematics of high range of motion activities of daily living. Journal of Orthopaedic Research, 2006, Vol. 24(4), pp. 770-781

\section{STATEMENTS AND DECLARATIONS}

[Instructions: Please check all applicable boxes and provide additional information as requested.]

\section{Conflict of Interest}

Potential conflict of interest exists:

We wish to draw the attention of the Editor to the following facts, which may be considered as potential conflicts of interest, and to significant financial contributions to this work:

The nature of potential conflict of interest is described below:

冈No conflict of interest exists.

We wish to confirm that there are no known conflicts of interest associated with this publication and there has been no significant financial support for this work that could have influenced its outcome.

\section{Funding}

冈Funding was received for this work.

All of the sources of funding for the work described in this publication are acknowledged below:

[List funding sources and their role in study design, data analysis, and result interpretation]

The authors wish to thank Universiti Malaysia Pahang (UMP) and Kementerian Pendidikan Malaysia (KPM) for the funding awarded to the author to complete this study under the national grant reference number: FRGS/1/2019/TK04/UMP/02/18 (FRGS) and RDU1901218 (UMP).

No funding was received for this work.

\section{Intellectual Property}

冈We confirm that we have given due consideration to the protection of intellectual property associated with this work and that there are no impediments to publication, including the timing of publication, with respect to intellectual property. In so doing we confirm that we have followed the regulations of our institutions concerning intellectual property.

\section{Authorship}

冈We confirm that the manuscript has been read and approved by all named authors.

冈We confirm that the order of authors listed in the manuscript has been approved by all named authors.

\section{Contact with the Editorial Office}

The Corresponding Author declared on the title page of the manuscript is:

\section{Ghassan Maan Salim}

冈This author submitted this manuscript using his/her account in editorial submission system.

\We understand that this Corresponding Author is the sole contact for the Editorial process (including the editorial submission system and direct communications with the office). He/she is responsible for communicating with the other authors about progress, submissions of revisions and final approval of proofs.

冈We confirm that the email address shown below is accessible by the Corresponding Author, is the address to which Corresponding Author's editorial submission system account is linked:

ghassan.m.a@ieee.org 
We the undersigned agree with all of the above.
Author's name (First, Last)
Signature
Date
1. Ghassan Maan Salim

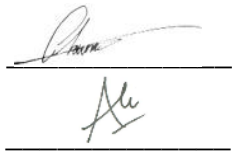
$\underline{17 / 12 / 2021}$
2. Mohd Anwar Zawawi
$\underline{17 / 12 / 2021}$ 